Recepción: 24/ 08 / 2018

Aceptación: 07 / 11 / 2018

Publicación: 03 / 12 / 2018

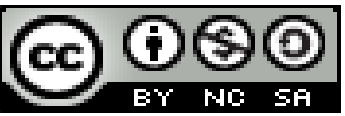

Ciencias de la educación

Artículo de revisión

\title{
La Investigación: acción como una estrategia pedagógica de relación entre lo académico y social
}
Research: action as a pedagogical strategy of relationship between academic and social

\section{Pesquisa: ação como estratégia pedagógica de relacionamento entre acadêmico e social}

\author{
Letty J. Saltos-Rodríguez ${ }^{\text {I }}$ \\ 1saltos@utm.edu.ec \\ Liliana del R. Loor-Salmon ${ }^{\text {II }}$ \\ 1loor@utm.edu.ec
}

\author{
Mayra M. Palma-Villavicencio ${ }^{\mathrm{III}}$ \\ mpalma@utm.edu.ec
}

\section{Correspondencia: 1saltos@utm.edu.ec}

\footnotetext{
${ }^{\text {I }}$ Magíster en Enseñanza del Idioma Inglés, Licenciada en Ciencias de la Educación Mención Inglés Francés, Docente del Instituto de Lenguas de la Universidad Técnica de Manabí, Portoviejo, Ecuador.

${ }^{\text {II }}$ Magíster en Docencia e Investigación Educativa, Doctora en Educación, Licenciada en Ciencias de la Educación Especialidad Inglés, Profesora de Segunda Enseñanza Idiomas y Lingüística Especialidad Inglés, Docente del Instituto de Lenguas de la Universidad Técnica de Manabí, Portoviejo, Ecuador.

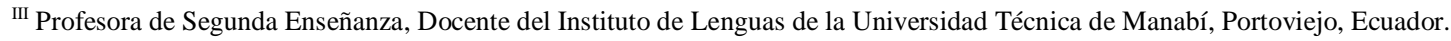




\title{
Resumen
}

La investigación-acción alude a una serie de estrategias metodológicas empleadas para el mejoramiento del sistema educativo y social, al pasar de lo mero social al compartir educacional. Atendiendo, esta posición en este artículo se presenta un estudio de la investigación - acción como una estrategia pedagógica de relación entre lo académico y social. La metodología fue de tipo descriptivo y documental. El corpus de la investigación la constituyó la revisión de fuentes bibliográficas y no bibliográficas La información se obtuvo a partir del registro, análisis e interpretación de las teorías relacionadas al tema en estudio. El enfoque epistémico fue de tipo hermenéutico, permitiendo el análisis de los contenidos de la información consultada de tipo académica o de divulgación, en formato físico o digital. Entre los resultados del análisis de la información, fue posible corroborar que las teorías relacionadas a la investigación acción dejo la exclusividad de uso para los temas sociales, convirtiéndose de gran importancia como estrategia pedagógica en la investigación académica.

Palabras clave: investigación - acción; estrategia pedagógica; investigación académica; investigación social.

\begin{abstract}
Action research refers to a series of methodological strategies used to improve the educational and social system, from the mere social to educational sharing. Attending, this position in this article presents a study of action research as a pedagogical strategy of relationship between academic and social. The methodology was descriptive and documentary. The corpus of the research was constituted by the review of bibliographic and non-bibliographic sources. The information was obtained from the registration, analysis and interpretation of the theories related to the subject under study. The epistemic approach was hermeneutical in nature, allowing the analysis of the contents of the information consulted for academic or dissemination purposes, in physical or digital format. Among the results of the analysis of the information, it was possible to corroborate that the theories related to action research left the exclusivity of use for social issues, becoming of great importance as a pedagogical strategy in academic research.
\end{abstract}

Key words: research - action; pedagogical strategy; academic research; social investigation. 


\section{Resumo}

A pesquisa-ação refere-se a uma série de estratégias metodológicas utilizadas para melhorar o sistema educacional e social, do mero compartilhamento social ao educacional. Atendendo, esta posição neste artigo apresenta um estudo de pesquisa-ação como uma estratégia pedagógica de relacionamento entre acadêmico e social. A metodologia foi descritiva e documental. O corpus da pesquisa foi constituído pela revisão de fontes bibliográficas e não bibliográficas, as quais foram obtidas a partir do registro, análise e interpretação das teorias relacionadas ao tema em estudo. A abordagem epistêmica foi de natureza hermenêutica, permitindo a análise do conteúdo das informações consultadas para fins acadêmicos ou de divulgação, em formato físico ou digital. Dentre os resultados da análise das informações, foi possível corroborar que as teorias relacionadas à pesquisa-ação deixaram a exclusividade de uso para questões sociais, tornando-se de grande importância como estratégia pedagógica na pesquisa acadêmica.

Palavras chave: pesquisa - ação; estratégia pedagógica; pesquisa acadêmica; pesquisa social.

\section{Introducción}

Existen múltiples definiciones, prácticas y sentidos, no habiendo criterios unificadores entre sí, relacionados al concepto de investigación -acción (I-A). Algunos la definen como investigación en el aula, profesorado investigador, investigación colaborativa, investigación participativa o investigación crítica, que designan modelos de I-A con características comunes. Para Latorre (2003), una primera definición que podemos utilizar, es aquella que describe una familia de actividades que realiza el profesorado en sus propias aulas, con fines tales como: el desarrollo curricular, su autodesarrollo profesional, la mejora de los programas educativos, los sistemas de planificación o la política de desarrollo. Estas actividades tienen en común la identificación de estrategias de acción que son implementadas y más tarde sometidas a observación, reflexión y replanteamiento. Se considera como un instrumento que genera cambio social y conocimiento educativo sobre la realidad social y/o educativa, proporciona autonomía y da poder a quienes la realizan.

Para Elliott (1993), la I-A se basa en el estudio de la realidad educativa que normalmente se contextualiza dentro del aula; es susceptible de llevarse a cabo por distintos miembros de la comunidad educativa (alumnos, maestros, padres, etc.). El mismo Latorre (2003), indica que en 
relación con la educación, se concibe como una indagación práctica realizada por el profesorado de forma colaborativa, que tiene la finalidad de mejorar su práctica educativa a través de ciclos de acción y reflexión, en la que sugiere avanzar hacia una educación innovadora fuera de los límites tradicionales y ser considerada un punto de partida como eje de formación, como objeto de reflexión y de construcción y, como objeto de transformación.

Por su parte, López (2005), sobre todo en relación a la construcción del conocimiento profesional que los profesores poseen y utilizan en sus tareas de enseñanza. Esta metodología representa una forma de entender la investigación integrando la actuación de los profesores a nivel participativo, colaborativo, democrático y crítico y también donde docentes y alumnos abordan colaborativamente sus problemas del aula. La Investigación-acción, indica Pérez (1990), puede constituir una formula privilegiada de renovación pedagógica, de perfeccionamiento del profesorado, de innovación educativa y de calidad de la educación. Con base a lo expuesto, se presenta en ese artículo un estudio de la investigación - acción como una estrategia pedagógica de relación entre lo académico y social.

\section{Desarrollo}

El psicólogo social alemán Kurt Lewin (1947), uno de los principales promotores de la I-A, afirmaba que a través de la investigación-acción era posible lograr de manera simultánea avances en el campo teórico y cambios sociales deseados. La investigación-acción es vista como una forma de investigación introspectiva combinada o colectiva. Su objeto es el mejoramiento de la racionalidad y la justicia en las prácticas educativas o sociales, pero al mismo tiempo ayudan a la comprensión de tales prácticas y de las situaciones en las que estas se producen.

Las teorías de la acción otorgan mucha importancia a las perspectivas comunes, dado que son prerrequisitos de las prácticas que se comparten durante el proceso de la investigación. No obstante, tal como lo indica Salgado, y col (2009), el objetivo de la investigación-acción no es el conocimiento práctico propiamente, pues este es apenas el comienzo. Lo importante es en realidad el "descubrimiento" que se hace y termina convirtiéndose en la base del proceso de concienciación y racionalización. Así, el docente se hace más consciente de algo y comprende mejor el proceso; es decir, se da cuenta de que la razón de ser de la investigación-acción es lograr 
la concienciación plena del estudiante con relación al proceso científico, tanto el proceso de producción de conocimiento como las experiencias de acción en concreto.

Para Elliott (1993), la I-A, se centra en el descubrimiento y resolución de los problemas a los que se enfrenta el profesorado en su práctica. Supone hacer una reflexión sobre los medios y los fines educativos, donde se integra la teoría con la práctica. Se trata del estudio de una situación social con el fin de mejorar la calidad de la acción dentro de la misma, es decir, realizar una acción sobre las acciones humanas y las situaciones sociales vividas por el profesorado que tiene como objetivo ampliar la comprensión de los docentes de sus problemas prácticos. Se pueden mencionar entre sus principales objetivos el de identificar y diagnosticar los problemas que surgen en al aula ante los enfoques de investigación-descubrimiento; desarrollar y comprobar hipótesis prácticas acerca de cómo pueden resolverse los problemas de la enseñanza previamente identificados; aclarar los objetivos, valores y principios, mediante la reflexión; elaborar actividades con objeto de modificar las circunstancias del grupo y habituarnos hacia la práctica de reflexión social desde la investigación.

Por su parte Kemmis (1992), explica que la I-A es una investigación sobre la práctica, realizada por y para los prácticos, en este caso por el profesorado. Los agentes involucrados en el proceso de investigación son participantes iguales, y deben implicarse en cada una de las etapas del proceso de investigación. Los principales beneficios de la I-A son la mejora y transformación de la práctica social para llegar a su comprensión, también acercarse a la realidad, y sobre todo crear un modelo de formación del profesorado que aúne los sistemas de investigación, acción, formación y creación de conocimiento.

Latorre (2003), indica que la I-A se caracteriza por su necesidad de integrar la acción. El mismo autor plantea que el foco de la investigación será el plan de acción para lograr el cambio o mejora de la práctica o propósito establecido, entendiendo que requiere una acción como parte integrante del mismo proceso de investigación y cuyo foco reside en los valores del profesional más que en las consideraciones metodológicas, en el sentido de que los profesionales investigan sus propias acciones.

Por su parte, Tesouro y col. (2007), señalan que entre los principales objetivos de la I-A educativa, se pueden mencionar el desarrollo curricular, el autodesarrollo profesional, la mejora 
de los programas educativos, los sistemas de planificación y la política de desarrollo, deduciendo la identificación común que tienen de estrategias de acción, que se observan, reflexionan y hasta se pueden cambiar, considerándose como un instrumento de cambio social y conocimiento educativo de la realidad social o educativa estudiada.

\section{Características de la Investigación Acción en la educación}

De acuerdo con Kemmis (1992), la estrategia de la investigación-acción presenta las siguientes características o rasgos más distintivos:

- Es participativa, pues los investigadores trabajan con el propósito de mejorar sus propias prácticas.

- Involucra un proceso sistemático de aprendizaje, que se orienta a la praxis.

- Comienza con pequeños ciclos de investigación (planificación, acción, observación y reflexión) que se van ampliando hacia problemas mayores. Del mismo modo, es iniciada por grupos pequeños de colaboradores para luego expandirse a grupos mayores gradualmente.

- La investigación sigue una línea introspectiva; es una especie de espiral que se desarrolla por ciclos cumpliendo las etapas de planificación, acción, observación y reflexión.

- Es de naturaleza colaborativa, pues se realiza en grupos.

- Busca crear comunidades científicas o académicas autocríticas, las cuales colaboran y participan en todas las etapas del proceso investigativo.

- Busca aproximar el objeto de la investigación y colaborar para el logro de los cambios sociales prácticos deseados.

- El proceso investigativo implica el registro, recopilación y análisis de los juicios propios, al igual que las reacciones e impresiones sobre las situaciones. Para esto requiere la redacción de un diario personal en el cual se exponen las reflexiones del investigador.

- Se le considera un proceso político, ya que implica cambios que pueden afectar a las personas.

Asimismo, entre otras particularidades la investigación-acción es descrita por otros autores como una alternativa a la investigación social tradicional, caracterizada por ser: 
- Práctica y pertinente, pues responde a problemas del entorno.

- Participativa y colaborativa, porque intervienen grupos de personas.

- Emancipadora por su enfoque simétrico no jerárquico.

- Interpretativa, porque asume las soluciones planteadas desde el punto de vista de los investigadores.

- Crítica, porque desde el principio se enfoca en el cambio.

En esencia, Kurt Lewin (1947), sugería que las tres características más importantes de la investigación acción moderna eran: su carácter participativo, su impulso democrático y su contribución simultánea al conocimiento en las ciencias sociales.

- Carácter participativo. Es una metodología que se ubica en el paradigma crítico-propositivo que, a diferencia del positivista o interpretativo requiere de la participación de los afectados por la preocupación temática estudiada. De este modo, los actores implicados se convierten en los protagonistas del proceso de construcción del conocimiento e intervención sobre la realidad. Las ideas cruciales en la obra de Lewin fueron las de decisión de grupo y compromiso con la mejora. El rasgo distintivo que adjudica este autor a la investigaciónacción es que aquellas personas que están afectadas por cambios planificados tienen una responsabilidad primaria en cuanto a decidir acerca de la orientación de una acción críticamente informada que parece susceptible de conducir a una mejora.

- Impulso democrático. Define el área sustantiva en la que el grupo decide centrar su estrategia de mejora, o sea que se refiere a la formulación de la naturaleza problemática de una determinada preocupación educativa. En el curso del proceso es habitual descubrir que la cuestión, preocupación o problema práctico que adquiere la forma de una preocupación temática compartida va cambiando a medida que se describe con mayor precisión y que la estrategia de acción provoca efectos.

- Contribución simultánea al conocimiento en las ciencias sociales. Algunos autores también conciben a la Investigación-acción como un camino para el desarrollo profesional. En esta línea se argumenta que es posible que los docentes (o también otros profesionales prácticos) mejoren sus habilidades o competencias para la ejecución de sus tareas en el campo donde se desempeñan. Dadas las características connaturales de los escenarios académicos, el 
educador encuentra ahí un espacio propicio para identificar temas y problemas inmediatos, comprenderlos, recrearlos y transformarlos. La vida académica facilita el perfeccionamiento de capacidades investigadoras para identificar problemas, observar, registrar, interpretar información, experimentar, planear, evaluar y escribir. Es por ello que, dentro de la investigación- acción se puede encontrar a la investigación-acción educativa como escenario y método potenciador del saber pedagógico.

El docente que se inicia en el ejercicio profesional pedagógico logra, según Restrepo, (2004), gracias a la investigación-acción una crítica a la propia práctica profesional que se conoce como primera fase o fase de construcción. En esta fase el docente vive un proceso de deconstrucción que va más allá de un auto examen de la práctica profesional y debe terminar con el encuentro de un saber pedagógico que justifique dicha práctica. El mismo autor, menciona una segunda fase de la investigación -acción educativa en la que la práctica profesional es reconstruida de manera que exista un diálogo entre teoría y práctica sustentado en un saber pedagógico subjetivo, individual y funcional. Finalmente se llega a la tercera etapa, en esta, se tiene lugar la validación de lo que hasta ahora ha sido ejecutado. La efectividad de la práctica alternativa y el grado de cumplimiento de los propósitos educativos planteados.

\section{Modelos de Investigación Acción}

Existen tres modelos o tipos de investigación-acción, los cuales corresponden a los distintos enfoques que hay de esta estrategia de investigación: la técnica, la práctica y la crítica emancipadora.

- Técnica. El propósito de este modelo de investigación-acción es lograr un mayor nivel de eficacia de las prácticas sociales. La estrategia es el fomento de la participación de los docentes en programas de investigación previamente diseñados por expertos o un equipo de trabajo. Los programas establecen los propósitos de la investigación y las pautas metodológicas que se deben seguir.

- Práctica. En este modelo de investigación-acción el cuerpo docente tiene un mayor protagonismo y autonomía. Son los investigadores (profesores) los encargados de seleccionar los problemas a investigar y de controlar el desarrollo del proyecto. Pueden incorporar a un 
investigador o consultor externo que colabore con el proceso investigativo y apoye la cooperación de quienes participan.

La investigación-acción práctica busca transformar la conciencia de los participantes y generar cambios en las prácticas sociales.

- Crítica emancipadora. Este modelo incorpora las ideas expuestas en la teoría crítica. Su trabajo está centrado en las prácticas educativas a través de las que intenta emancipar o liberar a los docentes de su trabajo cotidiano (rutinas, propósitos, creencias), así como establecer una vinculación entre su acción y el contexto social donde se desenvuelven. La investigaciónacción de tipo crítica emancipadora hace un esfuerzo por introducir cambios en la manera de trabajar (organización, contenidos, relaciones laborales).

\section{Metodología}

La metodología fue de tipo descriptivo y documental. Según Arias (2012), este tipo de investigación se caracteriza por describir la estructura o comportamiento de la variable estudiada tal como se presenta, en este caso particular se analizó la investigación - acción como una estrategia pedagógica de relación entre lo académico y social. Asimismo, permitió el registro, análisis e interpretación de las teorías manejadas en el estudio. El corpus de la investigación la constituyó la revisión de fuentes bibliográficas y no bibliográficas y el enfoque epistémico fue de tipo hermenéutico, permitiendo el análisis de los contenidos de la información teórica consultada.

\section{Conclusiones}

Se pudo constatar que el sistema de Investigación Acción, la cual surgió para dar respuesta a determinadas situaciones en las que intervenían grupos para intentar modificar acciones que requerían una toma de decisiones, y así poder tratar temas complejos donde no se podían utilizar los procedimientos experimentales clásicos conocidos hasta el momento, pero dado su carácter participativo, el impulso democrático y la contribución simultánea al cambio social y a la ciencia social se trasladó al campo educativo.

Es posible hacer constar, que el trabajo colaborativo que incita el uso de la investigación acción desde lo académico, en la cual los docentes colaboran por igual, ocupan sus roles y logran minimizar el individualismo, contribuyendo a realizar un trabajo de transformación educativa. 
Por otro lado, es un aliado del crecimiento investigativo en el campo de la educación socializando con los problemas de su entorno social, donde pueden participar los docentes, aun sin experiencia ni conocimiento del proyecto.

Es apreciable el hecho en la cual, las características de la Investigación - Acción para el campo de la educación, permiten un proceso sistemático de aprendizaje orientado a la práctica de los contenidos académicos tratados, que se orienta a la praxis. Dado que se inicia con pequeños ciclos de investigación (planificación, acción, observación y reflexión), los cuales se van ampliando hacia problemas mayores. Del mismo modo, es iniciada por grupos pequeños de colaboradores para luego expandirse a grupos mayores gradualmente, acción que favorece el quehacer practico - académico.

Siguiendo la propuesta de Lewin, el carácter participativo de la I-A, implica al sistema educativo el protagonismo de la construcción del conocimiento e intervención sobre su realidad social, dándolo un impulso democrático a la naturaleza problemática de una determinada preocupación educativa. Y de este modo, contribuir de forma simultánea al conocimiento en las ciencias sociales, dando la posibilidad a los docentes que intervienen en ella de mejorar sus habilidades o competencias para la ejecución de sus tareas en el campo donde se desempeñan. Es por ello que, dentro de la investigación- acción se puede encontrar a la investigación-acción educativa como escenario y método potenciador del saber pedagógico.

\section{Referencias Bibliográficas}

Arias, F. (2012). El proyecto de investigación. Introducción a la metodología científica. 6ta. Edición. Venezuela. Ediciones Fidias.

Elliott, J. (1993). El cambio educativo desde la investigación acción (1ª ed.). Madrid: Morata.

Kemmis, S. (1992). Cómo planificar la investigación-acción. Barcelona: Laertes.

Latorre, A. (2003). La investigación-acción. Conocer y cambiar la práctica educativa. Graó: Barcelona.

Lewin, Kurt (1947). "Action research and minority problems"; Journal of Social Issues 2 (4): 34 46. 
López Pastor, V (2005). Doce años de Investigación Acción en Educación Física. La importancia de las dinámicas colaborativas en la formación permanente del profesorado. El caso del grupo de trabajo internivelar de Segovia. Lecturas de Educación Física y Deportes, 90.

Pérez Serrano, G. (1990). Investigación-acción: aplicaciones al campo social y educativo. Madrid: Dykinson.

Restrepo, B. (2004). Una variante pedagógica de la investigación-acción educativa. OEI-Revista Iberoamericana de Educación.

Salgado, I. \& Silva-Peña, I. (2009). Desarrollo Profesional Docente en el Contexto de una Experiencia de Investigación Acción. Paradigma 30(2) 63-74

Tesouro, M.; Ribot, M.; Labian, I. y Guillamet, E.; Aguilera, A. (2007). Mejoremos los procesos de enseñanza-aprendizaje mediante la investigación-acción. Revista Iberoamericana de Educación. 